\title{
A Framework for Developing Pharmacotherapy for Agitation in Alzheimer's Disease: Recommendations of the ISCTM* Working Group
}

\author{
C. O'Gorman ${ }^{1}$, R. Khoury ${ }^{2}$, A. Anderson 3 , M. Carter ${ }^{4}$, F. DiCesare ${ }^{5}$, S. Dubé6, L. Ereshefsky ${ }^{7}$, G. Grossberg ${ }^{2}$,

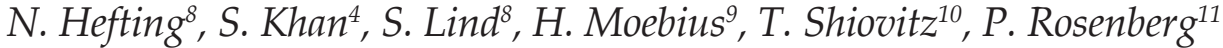

1. Axsome Therapeutics Inc., New York, NY, USA; 2. Department of Psychiatry and Behavioral Neuroscience, Saint Louis University School of Medicine, MO, USA; 3. Department of Psychiatry and Biobehavioral Sciences, University of California, Los Angeles, Los Angeles, CA, USA; 4. Pfizer Inc., Collegeville, PA, USA; 5. Leoben Research srl, Rome, Italy; 6. Avanir Pharmaceuticals Inc., Aliso Viejo, CA, USA; 7. Follow the Molecule LLC and Alliance for Early Phase, Marina del Rey, CA, USA; 8. H. Lundbeck A/S, Valby, Denmark; 9. moebius-consult GmbH, Baar, Switzerland; 10. California Neuroscience Research Medical Group Inc./CTSdatabase LLC., Sherman Oaks, CA, USA; 11. Department of Psychiatry and Behavioral Sciences, Johns Hopkins University School of Medicine, Baltimore, MD, USA. *ISCTM: International Society for CNS Clinical Trials and Methodology.

Corresponding Author: Cedric O'Gorman MD, 200 Broadway (3rd Floor), New York, NY 10038, USA, Email: cogorman@axsome.com

$\mathrm{D}$ ementia is characterized by a significant decline in one of several cognitive domains such as memory, language and executive function, affecting independence and representing a significant deterioration from a previous level of functioning (1). Alzheimer's Disease (AD) represents the most common form of dementia and contributes up to $70 \%$ of the almost 50 million dementia cases worldwide, a number that is projected to double in 20 years (2).

One or more behavioral and psychological symptoms of dementia (BPSD), also known as neuropsychiatric symptoms (NPS), including anxiety, depression, apathy, agitation and psychosis, occur in almost all dementia cases (3), and are associated with decreased functioning, increased caregiver burden, earlier care facility placement, faster disease progression and increased risk of death (4).

Agitation is one of the most challenging behavioral symptoms and is associated with a higher risk of institutionalization when compared to other BPSD (5), and a substantial economic burden (6).

Until recently, agitation in dementia was poorly defined and applied to heterogeneous patient populations. In 2015, an international group of experts under the auspices of the International Psychogeriatric Association (IPA) published a provisional consensus definition of agitation in patients with dementia (presented in table 1) to enhance communication regarding this condition and facilitate research in the field (7).

Despite its ubiquity in dementia, agitation is the clinical manifestation of disturbances in specific neuronal networks/circuits, which may vary according to the underlying etiology of dementia (8). A focus on agitation in $\mathrm{AD}$ subjects could result in the development of appropriately targeted pharmacotherapy, facilitate interpretation of clinical trial results and provide a clearer platform for regulatory decisions.
Most clinicians and guidelines recommend nonpharmacological interventions initially for the treatment of agitation in AD. These include reality orientation, behavioral management, environmental modification, cognitive stimulation, physical exercise and music (9). In addition, alternative treatments such as massage, aromatherapy and animal assisted-therapy have shown some benefit. When non-pharmacological approaches fail pharmacological interventions are frequently sought. While there are several approved medications for cognitive symptoms in $\mathrm{AD}$, no pharmacological treatment has yet been approved for agitation in AD.

In daily practice, clinicians attempt to fill this gap with off-label prescription of psychotropic drugs with concomitant limitations of efficacy, safety and tolerability. The prescribing of antipsychotics, especially in long-term care settings, is controversial and has been criticized. As a result, efforts have been taken in the United States (US) and the European Union (EU) to curb the use of antipsychotics in these settings (10). Notwithstanding their modest and variable efficacy in clinical trials of AD agitation (11,12), antipsychotics are prescribed despite being associated with increased mortality (resulting in the issuance of a boxed warning regarding their use in patients with dementia) (13, 14), an increased rate of cerebrovascular accidents, accelerated cognitive decline, gait disturbances, sedation and parkinsonism $(12,15,16)$. A recent meta analysis describes the trade-off between efficacy and safety of widely-used atypical antipsychotics in the treatment of behavioral and psychological symptoms of dementia (17). Similarly, benzodiazepine use may further impair cognition and adversely affect balance, thereby increasing the risk of falls $(18,19)$. Antidepressant drugs such as selective serotonergic reuptake inhibitors (SSRIs), widely used clinically, have shown limited efficacy in agitation studies. The CitAD trial found citalopram reduced agitation in patients with AD but was associated with 


\section{Table 1. Consensus Provisional Definition of Agitation in Cognitive Disorders}

A. The patient meets criteria for a cognitive impairment or dementia syndrome (e.g. AD, FTD, DLB, vascular dementia, other dementias, a pre-dementia cognitive impairment syndrome such as mild cognitive impairment or other cognitive disorder).

B. The patient exhibits at least one of the following behaviors that are associated with observed or inferred evidence of emotional distress (e.g. rapid changes in mood, irritability, outbursts). The behavior has been persistent or frequently recurrent for a minimum of two weeks and represents a change from the patient's usual behavior.

a. Excessive motor activity (examples include: pacing, rocking, gesturing, pointing fingers, restlessness, performing repetitious mannerisms).

b. Verbal aggression (e.g. yelling, speaking in an excessively loud voice, using profanity, screaming, shouting).

c. Physical aggression (e.g. grabbing, shoving, pushing, resisting, hitting others, kicking objects or people, scratching, biting, throwing objects, hitting self, slamming doors, tearing things, and destroying property).

C. Behaviors are severe enough to produce excess disability, which in the clinician's opinion is beyond that due to the cognitive impairment and including at least one of the following:

a. Significant impairment in interpersonal relationships.

b. Significant impairment in other aspects of social functioning.

c. Significant impairment in ability to perform or participate in daily living activities.

D. While co-morbid conditions may be present, the agitation is not attributable solely to another psychiatric disorder, suboptimal care conditions, medical condition, or the physiological effects of a substance.

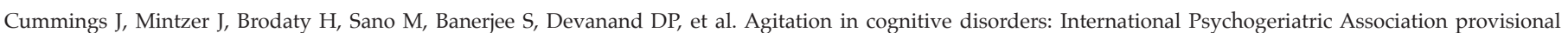
consensus clinical and research definition. Int Psychogeriatr. 2015;27(1):7-17. Table 1, Consensus provisional definition of agitation in cognitive disorders; p. 9.

worsening cognition and prolonged QTc interval. The most efficacious $30 \mathrm{mg}$ dose was also higher than the $20 \mathrm{mg}$ ceiling dose recommended for use in the elderly by the US Food and Drug Administration (FDA) (20). Regulators in the US and EU have thus encouraged the development of drug therapies addressing the unmet medical need to treat agitation in AD.

Appropriately designed clinical trials are required to ultimately fulfill this purpose.

\section{Objectives}

This paper is a product of the International Society for CNS Clinical Trials and Methodology (ISCTM), a multidisciplinary organization composed of persons in academia, industry, government, policy-making, and the public.

The goal of ISCTM is to develop a consensus amongst experts on a roadmap for optimal clinical trial methodology that may garner regulatory approval, and that can take the pharmacologic properties of different drug candidates into account. This paper summarizes the clinical development experience to date and the main learnings from past clinical trials. Commonly used assessment scales and promising biomarkers of agitation in $\mathrm{AD}$ are then reviewed. Current US and EU regulatory perspectives on trial methodology for agitation in $\mathrm{AD}$ are also discussed. Finally, the ISCTM proposes recommendations for future study designs in this field.

\section{Clinical Development Experience to date}

Double-blind, randomized, placebo-controlled studies of pharmacotherapies for agitation in dementia date back to the 1990s (21). Despite many failures, these studies brought increased awareness of major safety and tolerability limitations associated with the use of psychotropics, especially antipsychotics, in the elderly.

Successful drug development in the area has been challenged by the fluctuating and variable course of agitation symptoms as AD progresses. The patient population is relatively heterogenous in terms of cognitive impairment, functionality and behavioral symptoms throughout different stages of the disease (22). Furthermore, up until the recent development of a consensus definition of agitation in patients with cognitive impairment (7), the lack of a universally recognized definition made diagnosis and the conduct of clinical trials difficult. Care facility placement criteria also differed across trials which included a heterogeneous population of inpatients, nursing home patients and community-dwellers, complicating interpretation of outcomes.

More recently, there has been widespread adoption of the IPA provisional definition of agitation for diagnostic purposes. While most studies use the Mini-Mental State Examination (MMSE) to define AD severity, there is great variability in the inclusion of patients with mild, moderate and severe cognitive impairment across research trials. While it is an open question whether treatment approaches for agitation will vary across dementia severity, investigators should consider the option of stratifying randomization or performing subgroup analyses by dementia severity. 
Moreover, a recent review (23) found that treatment duration in published trials varies widely from 10 days to 12 months with a recent trend towards shorter trials. Shorter durations are theoretically preferred to limit elderly exposure to an investigational agent or even to placebo. A longer duration of assessment may be required to truly determine the benefit-risk profile of any pharmacological agent. Longer duration can be associated with loss of effect perhaps because of variability of symptoms over time. However, if an anti-agitation medicine could be used infrequently or occasionally to gain prompt control of an acute period of agitation, shorter durations of clinical testing may be appropriate. Shorter trials may be one solution but an alternate design might incorporate intermittent, as-needed treatment over a longer period of time. There is virtually no evidence to date to inform a choice to repeat or restart treatment at a later time.

Clinical trial methodologies in AD agitation also greatly differ in the extent to which use of concomitant psychiatric medications are permitted. Understandably, patients and caregivers are often wary of participation in a trial that necessitates washing out existing medications that may be providing some clinical benefit. When concomitant external medications are kept throughout a trial, researchers must carefully consider their effect on potential signal detection as well as safety and tolerability concerns due to combined medications in the elderly. Expert research groups have previously addressed the scientific and operational challenges related to designing clinical trials for agitation in dementia, with the aim of identifying better outcome measures $(24,25)$.

\section{Assessment Scales for Agitation in AD}

There is no standardized tool to assess agitation severity in clinical trials. Common instruments often and recently used include the Neuropsychiatric Inventory (26) and the Cohen-Mansfield Agitation Inventory (CMAI) (27).

The role of caregivers has also gained increasing attention and importance in observing clinical change in patients and determining outcomes in clinical trials. Proxy reporting bias can be an issue but caregiver report may be the only alternative given the clear challenges of interviewing a patient with $\mathrm{AD}$ who has both cognitive impairment and clinically important symptoms of agitation. Given the possible reporting bias associated with caregiver reports, an observational objective measure of the CMAI (CMAI-O) was recently developed and validated to be administered by a blinded researcher (28). Caregivers represent those who know their relative best and despite potential bias will be more keenly aware of changes in personality from their premorbid status. Accurate reporting can be improved with caregiver education regarding the signs and symptoms of agitation coupled with professional clinician oversight. Optimal scales as discussed below would ideally incorporate assessment from both caregiver and clinician.

The NPI has been widely used in clinical studies and originally included 10 sub-domains, including one for Agitation/ Aggression (NPI-AA). Further more specific questions are asked within each NPI subdomain if a caregiver/informant answers positively to the screening question (29). The sensitivity to change of the NPI has been debated. While it is not a continuous measure, it is common to assess the NPI using metric models which treat outcomes as continuous measures. However, since these measures lack certain possible values it is advisable to instead treat them as ordinal variables. Failure to properly adjust for the type of data being measured can increase false alarms and reduce statistical power (30). The NPI is likely more sensitive to change at the higher end of the scale. It is a limitation of the scale but fortunately is likely more sensitive at more clinically relevant scores.

The NPI-Q is an abbreviated version of the NPI which only includes screening questions, to facilitate its use in a clinical environment (31). More recently, a revised version of the NPI, the NPI-Clinician rating scale (NPI-C), which separates out agitation and aggression as separate domains, has been developed. It includes expanded domains and items, and a clinician-rating methodology (32). The agitation scale of the NPI was found to be highly correlated with the aggressiveness scale of the Behavioral Pathology in Alzheimer's disease (BEHAVEAD) (33), suggesting that these scales are capturing similar information. Given the fact that the NPI covers 10 domains that frequently overlap, there have been several efforts to develop subscales using composites of domains. For instance, Trepacz et al. (34), used a composite of four NPI domains (Agitation/Aggression, Disinhibition, Irritability, and Aberrant Motor Behavior) to measure agitation/aggression. However, a systematic review that adopted factor analyses of the NPI to describe agitation found low concordance between the studies. Furthermore, no study replicated the same cluster composition as the other (35).

From a regulatory perspective, it has been suggested that the NPI-AA may not align precisely with the IPA definition of agitation. Several limitations have been highlighted regarding the ability of the scale to adequately measure change in agitation in clinical trials. The instrument combines agitation and aggression, into a single score. Some stress that these behaviors are clinically distinct and should be measured separately. A decrease in the NPI-AA score over time may not convey whether agitation or aggression has improved or whether the severity or frequency of the symptoms has improved. However, the FDA has advised use of the NPI-C distinct domains of Agitation and Aggression as they include more items to improve ratings in milder and more severe symptoms and use clinician ratings as scores that incorporate data from patient and caregiver ratings with 
clinician judgment. In addition, they produce domain scores that encompass clinician's ratings on all items from each domain.

The BEHAVE-AD is mostly of historic interest and includes an aggressiveness component which measures symptoms of verbal outbursts, physical threats and violence, and agitation (non-verbal anger, negativity) (36). The BEHAVE-AD assesses 25 items on a four-point severity scale based on caregiver reports covering the past two weeks. Because caregiver report may introduce bias, the Empirical Behavioral Pathology in Alzheimer's Disease (E-BEHAVE-AD) clinician-rating scale was developed utilizing input from caregiver and patient interview and allowing clinicians to make a clinical judgment. Correlation between the scales was 0.51, with high inter-rater reliability seen among clinicians (37).

The CMAI is a 29-item caregiver-rating questionnaire (38). While historically developed for use in nursing homes, it has also been used outside this setting. This scale addresses the frequency of occurrence of symptoms across three categories: aggressive, physically nonaggressive, and verbally-agitated behaviors (27).

Physically-aggressive behaviors were less frequently seen than physically non-aggressive or verbally-agitated behaviors (39), but physically-aggressive behaviors were deemed more disruptive when they occurred (40). Reduction in the more severe and disruptive symptoms of agitation are of particular clinical importance. To what extent these domains are independent or clinically meaningful standalone entities in terms of treatment outcomes remains to be determined (41). There has been a recent trend towards using the CMAI as a primary endpoint in clinical trials, with the agreement of regulators. The CMAI demonstrated high internal consistency but only moderate interrater reliability (42) and showed good test-retest reliability over a onemonth period (39). The CMAI was correlated with the BEHAVE-AD in a study of residents of a long-term care facility (42). Studies using multiple instruments for assessing the efficacy of interventions for agitation typically lead to similar findings, but may be divergent from other change measures such as the Clinical Global Impression (CGI) (43). For example, the CMAI and NPI both showed statistically significant improvement with electroconvulsive therapy for treating agitation in dementia, but the CGI showed only slight changes (44). In the CitAD study, citalopram was effective for treating agitation in $\mathrm{AD}$ as demonstrated by the CMAI, however efficacy was not evidenced by the NPI-Agitation subscale, suggesting that the NPI subscale may be less sensitive to detecting statistically significant changes in agitation as compared to the CMAI (20). Agitation as a primary outcome may also be distinguished from psychosis. For instance, a recently published phase II study investigating pimavanserin for psychosis in AD reported statistically significant improvement in the NPI-nursing home psychosis subscale (delusions + hallucinations composite) but not in the NPI-nursing home Agitation/Aggression or CMAI (45).

\section{Biomarkers for Agitation Associated with AD}

A unique challenge for the development and use of diagnostic and response based biomarkers is their validation. Finding biomarkers has been challenging and the near-term likelihood of success is remote. There are none of the large scale genetic studies conducted that would be needed for an accurate genetic marker. Biomarker qualification is also a difficult regulatory process that has additional requirements beyond what is typically addressed in the scientific literature (46). There are currently no qualified biomarkers for the central nervous system listed by the FDA (47). The Biomarkers, EndpointS, and other Tools (BEST) Resource broadens the definition of biomarkers beyond the measurement of analytes in a biological matrix, encompassing other technologies such as sensors to evaluate movement patterns or electrophysiological measures (48). In 2011, the European Medicines Agency (EMA) qualified amyloid beta 1-42 and total-tau; positron-emissiontomography (PET) amyloid imaging (positive / negative) and low hippocampal volume (atrophy) as biomarkers for the purpose of enriching clinical trial populations in the early stages of $\mathrm{AD}$, however no specific association with agitation or BPSD was described at that time (49).

\section{Cerebrospinal fluid (CSF) biomarkers}

Bloniecki et al. showed a statistically significant correlation between CMAI agitation scores and elevated CSF total and phosphorylated tau levels, but not A $\beta 42$ levels in patients with AD (50). These findings are controversial as Skogseth and colleagues found no correlation between these CSF biomarkers in AD patients and agitation combined with delusions and hallucinations on the NPI (51). In another study, a weak but highly significant negative correlation was found between CSF A $\beta 42$ levels and aggressiveness on the BEHAVE-AD, but not with agitation as measured by the CMAI in patients with AD (52).

Moreover, no correlations were found between the CMAI and CSF cytokine (IL-6, 10 and sIL-1RII) levels (53).

\section{Plasma biomarkers}

Plasma levels of clusterin, a protein associated with apoptosis and clearance of cellular debris that binds to misfolded amyloid protein, and specifically the plasma/platelet clusterin ratio, appear promising (5457). Furthermore, in a longitudinal 2-year study, baseline high plasma $A \beta 42 / A \beta 40$ ratio significantly predicted the increase in aggression/agitation scores measured by the NPI, in ApoE4 carriers (57). 


\section{Imaging biomarkers}

\section{Structural neuroimaging}

Agitation measured by the NPI was significantly correlated with posterior cerebral atrophy, particularly in the right hemisphere $(58,59)$, atrophy of the frontal $(60)$, and the limbic $(60,61)$ structures, and higher temporal white matter hyperintensities (WMH) scores (59) on the magnetic resonance imaging (MRI) of patients with AD. These findings align with previous post-mortem autopsy evidence showing a correlation between agitation measured by the NPI and higher neurofibrillary tangles (NFT) density in bilateral orbito-frontal cortices and the anterior cingulate cortex (ACC) (62).

\section{Functional neuroimaging}

Single-photon emission computed tomographic (SPECT) scans measuring cerebral blood flow have demonstrated that hypoperfusion of the middle region of the right temporal gyrus significantly predicted the severity of aggression in patients with $\mathrm{AD}$, as measured by the BEHAVE-AD Aggressiveness subscale (63).

Sultzer and colleagues demonstrated a correlation between measures of agitation/disinhibition on the neurobehavioral rating scale (NRS) and hypometabolism in bilateral frontal and temporal lobes on [18F] fluorodeoxyglucose (FDG) positron emission tomography (PET) (64). Additionally, compared to both young and older healthy controls, patients with mild AD and agitation (measured by the NPI) had significantly increased left amygdala activation to familiar neutral (non-emotional) stimuli on functional MRI studies (65).

In a magnetic resonance spectroscopy (MRS) study of patients with $\mathrm{AD}$, agitation scores (by CMAI) were shown to correlate negatively with the $\mathrm{N}$-acetylaspartateto-creatine (NAA/Cr) ratio over the left posterior cingulate gyrus. NAA/ $\mathrm{Cr}$ is a measure of metabolic state and neuronal density (66).

\section{Novel biomarkers}

\section{Sensors}

In a recent review, actigraphy shows evidence of correlation with incidence of agitation and aggression; however, multimodal sensing has not been fully evaluated for this purpose (67). Deep machine learning algorithms in development may well result in significant strides in the use of multimodal sensing technologies as objective measures of agitation and provide evidence of treatment effects.

\section{Biomarkers of the neurotransmitter systems}

Neurochemical markers and genetic polymorphisms of the neurotransmitter system have been gaining attention in the field of neuropsychiatric disorders; and are interesting as potential diagnostic, prognostic, predictive, and / or monitoring markers of agitation in AD. Measures of serotonin dysfunction, including receptor and transporter polymorphisms, and low central activity also have potential for better understanding NPS mechanisms (68).

\section{A Regulatory History}

Regulator views on the appropriateness of agitation in $\mathrm{AD}$ as a legitimate target for drug development have significantly evolved during the last 20 years. In March 2000, the FDA raised the issue of agitation being an illdefined syndrome which cannot be endorsed as a distinct development indication, as it may yield artificially narrow labeling claims and inadequate drug use (69, 70). However, following the recognition by the broad scientific community of agitation as a distinct, clinically identifiable syndrome (7), the US FDA (71), the EU-EMA (72-74), and the Japan Pharmaceuticals and Medical Devices Agency-PMDA (75) currently acknowledge agitation in $\mathrm{AD}$ as a prevalent and serious condition with a valid pressing need for treatment development.

There exist regulatory processes, including breakthrough designation and PRIME, whereby commitment has been demonstrated on the part of regulators to expedite development and review of candidate investigational products within areas of significant unmet need, including this one. This is evidenced in the US by a host of recent Fast Track Designation grants for various new chemical entities, such as: TNX-102 SL (cyclobenzaprine HCl; July 2018), AXS-05 (dextromethorphan/bupropion modulated delivery tablet); May 2017) (76), AVP-786 (deuterateddextromethorphan and quinidine; November 2015) (77), and ELND005 (scyllo-inositol; July 2013) (78). For other regulatory jurisdictions, formal guidelines exist (e.g., EMA AD guideline, 2018) attesting to their interest in agitation as a target for drug development.

Generally, regulators recommend that late stage confirmatory studies conducted to support New Drug Application (NDA) or Marketing Authorization Application (MAA) submission in agitation in AD include patients diagnosed with probable AD with persistent symptoms of agitation treated in a randomized, double-blind, placebo-controlled fashion comparing safety and efficacy of the investigational agent versus placebo $(71,72)$. In line with the International Council for Harmonization of Technical Requirements for Registration of Pharmaceuticals for Human Use ( $\mathrm{ICH})$ E1 guidelines (79), a one-year open label extension safety and tolerability study with at least 100 patients 
evaluated should be conducted in case chronic use of the investigational agent is foreseen. The duration of the open label extension studies depends on the safety data and the expected safety profile of the drug in the $\mathrm{AD}$ population.

Several assessment tools for agitation are used clinically and in research, including the CMAI, NPI, NPI-C and BEHAVE-AD (71-75). Of these currently only the CMAI and the NPI-C appear to have been accepted as meeting regulatory requirements for study endpoints which could support regulatory approval. There is also a general agreement that key secondary outcome measures should target a separate domain (i.e. measures of functional capacity or caregiver distress), rather than replicating the key symptom domain assessed by the primary measure (80).

Furthermore, the registration landscape is evolving to include the utilization of novel digital endpoints and clinical outcome assessments (such as clinical, caregiver and patient functional outcome measures). It is generally agreed among regulators that endpoints should be patient-focused in that one should consider functioning in daily activities as well as symptom severity alone as meaningful outcomes. The applicability of these nascent programs should be considered for drug developers interested in entering this space with early engagement with global health authorities.

It is important that sponsors engage regulators early and often within the development process (ideally, prior to first-in-human study initiation and especially prior to commencing pivotal trials intended to support marketing applications) to gain alignment on study design elements to support registration. Such interactions allow sponsors the opportunity to further understand regulators' thinking and to work collaboratively to identify the best path forward for a specific investigational product development program.

\section{Design for Future Trials of Agitation in AD: Recommendations of the ISCTM Working Group}

\section{Study Population}

An appropriately broad yet representative study population of $\mathrm{AD}$ patients with agitation as defined by the IPA consensus definition should be enrolled. The study population should approximate the patient population that would ultimately be prescribed any approved medication, and should be clearly defined by inclusion and exclusion criteria. Consideration must be given to the stage of dementia (mild/moderate/severe), severity of agitation/aggression, the heterogeneity of presentation of agitation symptoms across multiple domains and the care settings (community-dwellers/ inpatients/nursing home patients). In addition to pooled analyses, patient stratification and clustering analyses for subgroup identification (taking into account the aforementioned parameters) are necessary for a more accurate evaluation of outcomes of potential interventions.

\section{Duration and set-up of trials}

The duration of a trial largely depends on the intended use of the drug (eg. frequency and chronicity) and how long it may take the drug to elicit an effect. Efficacy trials are generally not longer than 12 weeks, including the titration period (if needed). For acute indications, a rapid onset of action needs to be demonstrated. For prevention or treatment of agitation over weeks to months, the length of the trial would need to be appropriate to evaluate the risk-benefit of longer term use. Efforts should generally be made to reduce the time required for elderly subjects to be exposed to investigational agents or placebo/ controls in clinical trials.

\section{Recommended assessment tools and endpoints}

Assessment tools need to account for the perspectives of the patient, caregiver and clinician for a holisitic and reliable determination of treatment response. This will require educating and training caregivers to provide well-informed and objective assessments. Validated mobile data collection and evaluation tools have recently emerged as reliable markers for patient status and treatment effects; their use is thus encouraged in clinical studies. In this regard a recent paper reported activity counts (measured by wrist actigraphy) as being well correlated with agitation rating scales in a nursing home demented population $(81,82)$.

\section{Primary endpoints}

Change from baseline in the score on at least one widely used and validated clinician-rated assessment tool such as the NPI-C or CMAI should be used as a primary endpoint in a regulatory trial. The CMAI seems to be the most appropriate tool as it is the most consistent with the symptoms comprised in the IPA agitation definition.

\section{Secondary endpoints}

They should assess aspects of the drug effects that are unrelated to the primary symptoms of the disease. Examples include changes in the Clinical Global Impression Severity scale (CGI-S) that reflects the patient's overall improvement during the trial. Scales measuring the impact of the intervention on caregiver distress can also be incorporated, and a caregiver global impression scale is of great clinical importance given that the caregiver is presumably the one who spends 
the most time with the patient. It is essential however, in the opinion of this group and regulators, that any intervention first and foremost ameliorate symptoms for the patient independent of measures of caregiver distress.

\section{Exploratory endpoints}

Collection of real-time data on agitation symptoms from both patients (e.g. actigraphy) or near realtime data from caregivers (e.g. through smart phone diary applications) may augment or replace exisitng retrospective rating scales. Exploratory endpoints that involve such technologies are encouraged.

\section{Concomitant Treatment}

\section{Concomitant use of cognitive enhancers (cholinesterase inhibitors and/or memantine)}

This practice is generally acceptable in this population, provided there are no significant drug-drug interactions and a period of dosing stability is achieved, often two or three months, prior to enrollment. It may be important to stratify the patient population by concomitant centrally-acting medications (cognitive enhancers and other psychotropics [see below]) to further delineate observed treatment effects and safety findings. The data on whether cognitive enhancers have efficacy against agitation in AD are quite mixed and often null, and thus the effect size seems small at best, so allowing the use of cognitive enhancers is not likely to markedly bias trial results.

\section{Concomitant use of other psychotropics}

Use of other psychotropics, such as antipsychotics, antidepressants or benzodiazepines, may confound the evaluation of beneficial effects of a novel agitation treatment. Additionally, the mechanisms of action of investigational agents may require that certain medications are prohibited not only to isolate any potential efficacy signal but also to avoid overstimulation of shared neurobiological pathways. However, we recommend allowing inclusion of stable doses of carefully selected psychotropics when clinically indicated, provided they do not significantly interfere with safety or the interpretation of study results. Judicious allowance of a rescue medication, such as low doses of lorazepam, may also be required in clinical trials with this population. Use of concomitant medication and rescue interventions may be addressed in the statistical analysis (83).

\section{Disclosures to participants}

Provide basic information on dealing with agitation equally to all participants and their caregivers in these studies. Any social and behavioral treatments or approaches that were initiated a reasonable time period (e.g. 4 weeks) prior to randomization can be continued at the same frequency. Allowance of initiation of psychosocial or cognitive behavior therapy after enrollment should be carefully considered as it may interfere with the trial objectives and interpretation of results.

\section{Reduction of placebo response and treatment non-adherence}

As in many areas of research, particularly neuropsychiatric research, non-adherence and placebo response challenge the interpretation of results. Educating caregivers, tempering expectations, checking for duplicate subjects, assessing pharmacokinetic adherence levels, considering technology-based interventions to enhance adherence, particularly in phase II trials, may provide valuable insights and help mitigate the effects of these confounders on the study analyses. Utilization of independent external patient adjudication techniques to ensure the enrollment of patients with a correct diagnosis and appropriate level of symptom severity can also be invaluable. Regulators should be involved early in the course of development if novel designs such as the Sequential Parallel Comparison Design (SPCD) or placebo lead-in are intended to be included in the confirmatory program.

\section{Biomarkers}

Research correlating biomarkers and agitation in $\mathrm{AD}$ is scarce. Published studies are underpowered by their small sample size and the heterogeneity of instruments used to assess for agitation, in this heterogeneous population. No CSF, plasma or imaging biomarker can be recommended yet to be used in future clinical trials. A greater collaboration in the 'preclinical space' between the researchers, sponsors and regulators is encouraged to overcome the considerable challenges associated with biomarker validation. Exploratory measurement of more than one biomarker in a study, i.e., imaging modality plus tau, and/or inflammatory markers and or neurochemical measures is also encouraged to detect 'convergent' signals and help guide future research and knowledge. 


\section{Conclusion}

ISCTM is a multi-disciplinary independent organization, devoted to promoting advances that address strategic clinical, regulatory, methodological and policy challenges that arise in the development and use of CNS therapeutic agents. This ISCTM working group on agitation in Alzheimer's Disease sought input, opinion and expertise from a broad range of individuals from academia, industry, clinical and regulatory agencies to best inform consensus recommendations. These recommendations intend to support the design of clinical development programs aimed at finding safe and effective treatments for the millions of individuals living with agitation associated with Alzheimer's disease.

Dedication: This article is dedicated to the memory of Dr. Brendon Binneman who contributed generously of his time and expertise in the early stages of this project.

Acknowledgement: The authors wish to thank Drs. Tiffany Farchione, Valentina Mantua and Wen-Hung Chen for providing regulatory perspective. They also wish to thank Crystin Gloude for medical writing assistance.

Conflict of interests: COG is an employee of Axsome Therapeutics, Inc. MC and SK are full-time employees of Pfizer Inc. SD is a full-time employee of Avanir Pharmaceuticals, Inc. LE holds chief scientific officer positions at Hassman Reseach Institute and CNS Network. GG is a consultant for Acadia, Alkahest, Allergen, Avanir, Axovant, Bioxcel, GE, Genentech, Lundbeck, Novartis, Otsuka, Roche and Takeda; has received research support from Janssen, Roche and the National Institute of Aging (NIA); has been on safety monitoring committees for EryDel, Merck and Newron; and is on a speaker bureau for Acadia. NH and SL are full-time employees of Lundbeck. HM is co-founder and acting CMO at Exciva $\mathrm{GmbH}$. PR has received support for this work through grants 5R01AG054771 and 5R01AG050515-03 from the National Institute of Aging (NIA) and has received support for other research from Eli Lilly, the Alzheimer's Association and the NIA. No other disclosures were reported.

Ethical standards: The authors followed the ethical guidelines of the Journal for this manuscript.

Open Access: This article is distributed under the terms of the Creative Commons Attribution 4.0 International License (http:// creativecommons.org/ licenses/by/4.0/), which permits use, duplication, adaptation, distribution and reproduction in any medium or format, as long as you give appropriate credit to the original author(s) and the source, provide a link to the Creative Commons license and indicate if changes were made.

\section{References}

1. American Psychiatric Association, Diagnostic And Statistical Manual Of Mental Disorders. 5th ed. 2013, Washington DC: American Psychiatric Association Publishing

2. World Health Organization. Dementia [Internet] 2017 Dec 12 [cited 2018 May 12]; Available from: http: / / www.who.int/ news-room/fact-sheets/detail/ dementia.

3. Gauthier, S., et al., Management of behavioral problems in Alzheimer's disease. Int Psychogeriatr, 2010. 22(3): p. 346-72.

4. Kales, H.C., L.N. Gitlin, and C.G. Lyketsos, Assessment and management of behavioral and psychological symptoms of dementia. BMJ, 2015. 350: p. h369.

5. Okura, T., et al., Neuropsychiatric symptoms and the risk of institutionalization and death: the aging, demographics, and memory study. Journal of the American Geriatrics Society, 2011. 59(3): p. 473-481.

6. Morris, S., et al., Monetary costs of agitation in older adults with Alzheimer's disease in the UK: prospective cohort study. BMJ Open, 2015. 5(3): p. e007382.

7. Cummings, J., et al., Agitation in cognitive disorders: International Psychogeriatric Association provisional consensus clinical and research definition. Int Psychogeriatr, 2015. 27(1): p. 7-17.

8. Rosenberg, P.B., M.A. Nowrangi, and C.G. Lyketsos, Neuropsychiatric symptoms in Alzheimer's disease: What might be associated brain circuits? Mol Aspects Med, 2015. 43-44: p. 25-37.

9. King JB, Jones KG, Goldberg E, et al. Increased Functional Connectivity After Listening to Favored Music in Adults With Alzheimer Dementia. J Prev
Alzheimers Dis. 2019;6(1):56-62.

10. Senators Blumenthal, Kohl, Grassley Seek To Protect The Elderly And Taxpayers From Abusive Overprescribing Of Antipsychotics. 2012.

11. Ballard, C. and J. Waite, The effectiveness of atypical antipsychotics for the treatment of aggression and psychosis in Alzheimer's disease. Cochrane Database Syst Rev, 2006(1): p. Cd003476.

12. Schneider, L.S., K. Dagerman, and P.S. Insel, Efficacy and adverse effects of atypical antipsychotics for dementia: meta-analysis of randomized, placebocontrolled trials. Am J Geriatr Psychiatry, 2006. 14(3): p. 191-210.

13. Lenzer, J., FDA warns about using antipsychotic drugs for dementia. BMJ (Clinical research ed.), 2005. 330(7497): p. 922-922.

14. Psychiatric News, «FDA Extends Black-Box Warning to All Antipsychotics.». 2008. 43, 1-27 DOI: / / doi.org/10.1176/pn.43.14.0001.

15. Schneider, L.S., et al., Effectiveness of atypical antipsychotic drugs in patients with Alzheimer's disease. N Engl J Med, 2006. 355(15): p. 1525-38.

16. Ballard, C.G., et al., Management of agitation and aggression associated with Alzheimer disease. Nat Rev Neurol, 2009. 5(5): p. 245-55.

17. Yunusa, I., et al., Assessment of Reported Comparative Effectiveness and Safety of Atypical Antipsychotics in the Treatment of Behavioral and Psychological Symptoms of Dementia: A Network Meta-analysis. JAMA Netw Open, 2019. 2(3): p. e190828.

18. Diaz-Gutierrez, M.J., et al., Relationship between the use of benzodiazepines and falls in older adults: A systematic review. Maturitas, 2017. 101: p. 17-22.

19. Defrancesco, M., et al., Use of Benzodiazepines in Alzheimer's Disease: A Systematic Review of Literature. The International Journal of Neuropsychopharmacology, 2015. 18(10): p. pyv055-pyv055.

20. Porsteinsson, A.P., et al., Effect of citalopram on agitation in Alzheimer disease: the CitAD randomized clinical trial. JAMA, 2014. 311(7): p. 682-691.

21. Schneider, L.S., V.E. Pollock, and S.A. Lyness, A metaanalysis of controlled trials of neuroleptic treatment in dementia. J Am Geriatr Soc, 1990. 38(5): p 553-63.

22. Schneider, L.S., et al., Heterogeneity of Treatment Response to Citalopram for Patients With Alzheimer's Disease With Aggression or Agitation: The CitAD Randomized Clinical Trial. Am J Psychiatry, 2016. 173(5): p. 465-72.

23. Dube, S., J.T. Megerian, and R. Malamut, A Literature Review of Methodologies Used in Randomized Clinical Trials of Agitation in Alzheimer's Disease. J Prev Alzheimers Dis, 2018. 5(2): p. 120-133.

24. Sano M, Soto M, Carrillo M, et al. Identifying Better Outcome Measures to Improve Treatment of Agitation in Dementia: A Report from the EU/US/ CTAD Task Force. J Prev Alzheimers Dis. 2018;5(2):98-102.

25. Siegfried K, Legrand V. Studies of Agitation / Aggression in Alzheimer's Disease - Scientific and Operational Challenges. J Prev Alzheimers Dis. 2015;2(3):157-159.

26. Cummings, J.L., The Neuropsychiatric Inventory Assessing psychopathology in dementia patients. Neurology, 1997. 48(5 Suppl 6): p. 10S-16S.

27. Cohen-Mansfield, J., M.S. Marx, and A.S. Rosenthal, A description of agitation in a nursing home. Journal of gerontology, 1989. 44(3): p. M77-M84.

28. Griffiths AW, Albertyn CP, Burnley NL, et al. Validation of the CohenMansfield Agitation Inventory Observational (CMAI-O) tool. Int Psychogeriatr. 2020;32(1):75-85.

29. Cummings, J.L., et al., The Neuropsychiatric Inventory comprehensive assessment of psychopathology in dementia. Neurology, 1994. 44(12): p. 23082308 .

30. Liddell TM, Kruschke. Analyzing ordinal data with metric models: What could possibly go wrong? J Exp Soc Psychol. 2018; 79:328-348.

31. Kaufer, D.I., et al., Validation of the NPI-Q, a brief clinical form of the Neuropsychiatric Inventory. The Journal of Neuropsychiatry and Clinical Neurosciences, 2000. 12(2): p. 233-239.

32. De Medeiros, K., et al., The Neuropsychiatric Inventory-Clinician rating scale (NPI-C): reliability and validity of a revised assessment of neuropsychiatric symptoms in dementia. Int Psychogeriatr, 2010. 22(6): p. 984-94.

33. Reisberg, B., et al., Behavioral symptoms in Alzheimer's disease: phenomenology and treatment. The Journal of Clinical Psychiatry, 1987.

34. Trzepacz, P.T., et al., Subscale validation of the neuropsychiatric inventory questionnaire: comparison of Alzheimer's disease neuroimaging initiative and national Alzheimer's coordinating center cohorts. The American Journal of Geriatric Psychiatry, 2013. 21(7): p. 607-622.

35. Canevelli, M., et al., Behavioral and psychological subsyndromes in Alzheimer's disease using the Neuropsychiatric Inventory. International Journal of Geriatric Psychiatry, 2013. 28(8): p. 795-803.

36. Reisberg, B., S.R. Auer, and I.M. Monteiro, Behavioral pathology in Alzheimer's disease (BEHAVE-AD) rating scale. International Psychogeriatrics, 1997. 8(S3): p. 301-308.

37. Auer, S.R., I.M. Monteiro, and B. Reisberg, The empirical behavioral pathology in Alzheimer's disease (E-BEHAVE-AD) rating scale. International Psychogeriatrics, 1996. 8(2): p. 247-266.

38. Cohen-Mansfield, J. and N. Billig, Agitated behaviors in the elderly: I. A conceptual review. Journal of the American Geriatrics Society, 1986. 34(10): p 711-721.

39. Koss, E., et al., Assessing patterns of agitation in Alzheimer's disease patients with the Cohen-Mansfield Agitation Inventory. The Alzheimer's Disease Cooperative Study. Alzheimer Disease and Associated Disorders, 1997. 11: p. 
S45-50.

40. Cohen-Mansfield, J., Agitated behavior in persons with dementia: the relationship between type of behavior, its frequency, and its disruptiveness. Journal of Psychiatric Research, 2008. 43(1): p. 64-69.

41. Rabinowitz, J., et al., Factor analysis of the Cohen-Mansfield Agitation Inventory in three large samples of nursing home patients with dementia and behavioral disturbance. Am J Geriatr Psychiatry, 2005. 13(11): p. 991-8.

42. Finkel, S.I., J.S. Lyons, and R.L. Anderson, Reliability and validity of the Cohen-Mansfield agitation inventory in institutionalized elderly. International Journal of Geriatric Psychiatry, 1992. 7(7): p. 487-490.

43. Ijaopo, E., Dementia-related agitation: a review of non-pharmacological interventions and analysis of risks and benefits of pharmacotherapy. Translational Psychiatry, 2017. 7(10): p. e1250.

44. Acharya, D., et al., Safety and utility of acute electroconvulsive therapy for agitation and aggression in dementia. International Journal of Geriatric Psychiatry, 2015. 30(3): p. 265-273.

45. Ballard, C., et al., Evaluation of the safety, tolerability, and efficacy of pimavanserin versus placebo in patients with Alzheimer's disease psychosis: a phase 2, randomised, placebo-controlled, double-blind study. The Lancet Neurology, 2018. 17(3): p. 213-222.

46. U.S. Department of Health and Human Services, Food and Drug Administration Center for Drug Evaluation and Research (CDER) Services. Guidance for Industry and FDA staff- Qualification Process for Drug Development Tools. 2014 11/13/2018].

47. U.S. Food and Drug Administration. List of Qualified Biomarkers 09/11/2018 11/13/2018]; Available from: https://www.fda.gov/ Drugs / Development ApprovalProcess / DrugDevelopmentTools QualificationProgram/BiomarkerQualificationProgram/ucm535383.htm.

48. U.S. Food and Drug Administration. The best resource: Harmonizing biomarker terminology 11/13/2018; Available from: https:/ / www.fda.gov/downloads / drugs / developmentapprovalprocess / drugdevelopmenttoolsqualificationprogram/ucm510443.pdf..

49. European Medicines Agency-EMA, Qualification of novel methodologies for medicine development. 2011 [cited 2019 04/06/2019]; Available from: https: / / www.ema.europa.eu/en/human-regulatory/research-development/ scientific-advice-protocol-assistance / qualification-novel-methodologiesmedicine-development.

50. Bloniecki, V., et al., Agitation in dementia: relation to core cerebrospinal fluid biomarker levels. Dement Geriatr Cogn Dis Extra, 2014. 4(2): p. 335-43.

51. Skogseth, R., et al., Neuropsychiatric Correlates of Cerebrospinal Fluid Biomarkers in Alzheimer's Disease. Dementia and Geriatric Cognitive Disorders, 2008. 25(6): p. 559-563.

52. Engelborghs, S., et al., Neuropsychological and behavioural correlates of CSF biomarkers in dementia. Neurochemistry international, 2006. 48(4): p. 286-295.

53. Holmgren, S., et al., Neuropsychiatric symptoms in dementia-A role for neuroinflammation? Brain Research Bulletin, 2014. 108: p. 88-93.

54. Silajdzic, E., et al., No diagnostic value of plasma clusterin in Alzheimer's disease. PLoS One, 2012. 7(11): p. e50237.

55. Xing, Y.Y., et al., Blood clusterin levels, rs9331888 polymorphism, and the risk of Alzheimer's disease. J Alzheimers Dis, 2012. 29(3): p. 515-9.

56. Mukaetova-Ladinska, E.B., et al., Plasma and platelet clusterin ratio is altered in Alzheimer's disease patients with distinct neuropsychiatric symptoms: findings from a pilot study. Int J Geriatr Psychiatry, 2015. 30(4): p. 368-75.

57. Hsu, J.-L., et al., The clinical significance of plasma clusterin and $A \beta$ in the longitudinal follow-up of patients with Alzheimer's disease. Alzheimer's Research \& Therapy, 2017. 9: p. 91

58. Hsu, J.L., et al., Plasma biomarkers are associated with agitation and regional brain atrophy in Alzheimer's disease. Sci Rep, 2017. 7(1): p. 5035.

59. Hsu, J.L., et al., Posterior Atrophy and Medial Temporal Atrophy Scores Are Associated with Different Symptoms in Patients with Alzheimer's Disease and Mild Cognitive Impairment. PLoS One, 2015. 10(9): p. e0137121.

60. Trzepacz, P.T., et al., Frontolimbic atrophy is associated with agitation and aggression in mild cognitive impairment and Alzheimer's disease. Alzheimer's \& Dementia : the journal of the Alzheimer's Association, 2013. 9(5 0): p. S95-S104.e1
61. Bruen, P.D., et al., Neuroanatomical correlates of neuropsychiatric symptoms in Alzheimer's disease. Brain, 2008. 131(9): p. 2455-2463.

62. Tekin, S., et al., Orbitofrontal and anterior cingulate cortex neurofibrillary tangle burden is associated with agitation in Alzheimer disease. Ann Neurol, 2001. 49(3): p. 355-61.

63. Lanctot, K.L., et al., Medial temporal hypoperfusion and aggression in Alzheimer disease. Arch Neurol, 2004. 61(11): p. 1731-7.

64. Sultzer, D.L., et al., The relationship between psychiatric symptoms and regional cortical metabolism in Alzheimer's disease. J Neuropsychiatry Clin Neurosci, 1995. 7(4): p. 476-84.

65. Wright, C.I., et al., A functional magnetic resonance imaging study of amygdala responses to human faces in aging and mild Alzheimer's disease. Biol Psychiatry, 2007. 62(12): p. 1388-95.

66. Tsai, C.F., et al., Differences in brain metabolism associated with agitation and depression in Alzheimer's disease. East Asian Arch Psychiatry, 2013. 23(3): p. 86-90.

67. Khan, S.S., et al., Detecting agitation and aggression in people with dementia using sensors-A systematic review. Alzheimers Dement, 2018.

68. Ruthirakuhan, M., et al., Biomarkers of agitation and aggression in Alzheimer's disease: A systematic review. Alzheimer's \& Dementia: The Journal of the Alzheimer's Association, 2018. 14(10): p. 1344-1376.

69. Laughren, T., Regulatory Issues on Behavioral and Psychological Symptoms of Dementia in the United States. International Psychogeriatrics, 2000. 12(S1): p. 331-336.

70. Laughren, T., A regulatory perspective on psychiatric syndromes in Alzheimer disease. Am J Geriatr Psychiatry, 2001. 9(4): p. 340-5.

71. Farchione T., Regulatory Discussion: Agitation in Alzheimer 's Disease FDA Perspective, in ISCTM Autumn Conference. 2017.

72. Mantua V., Agitation in Alzheimer's Disease: A Regulatory Viewpoint, in ISCTM Autumn Conference. 2017

73. European Medicines Agency, Clinical investigation of medicines for the treatment of Alzheimer's disease. 2018 12/2/2018]; Available from: https:// www.ema.europa.eu/en/clinical-investigation-medicines-treatmentalzheimers-disease.

74. Pani L., The place for treatments of associated neuropsychiatric and other symptoms, in European Medicines Agency workshop on the clinical investigation of medicines for the treatment of Alzheimer's disease. 2014.

75. Kamata A., Drug Development for Behavioral and Psychological Symptoms of Dementia in Japan in European Medicines Agency workshop on the clinical investigation of medicines for the treatment of Alzheimer's disease 2014

76. Axsome Therapeutics, Axsome Therapeutics Receives FDA Fast Track Designation For AXS-05 For Alzheimer's Disease Agitation. 2017.

77. Avanir Pharmaceuticals, A., Avanir Pharmaceuticals Announces Initiation of Phase III Trial of AVP-786 for Agitation in Patients with Alzheimer's Disease. 2015.

78. Transition Therapeutics, FDA Grant Fast Track Designation to ELND005 for the Treatment of Neuropsychiatric Symptoms in Alzheimer's Disease. 2013.

79. ICH Expert Working Group, Harmonised Tripartite Guideline E1-The extent of population exposure to assess clinical safety for drugs intended for long-term treatment of non-life-threatening conditions E1 in International conference on harmonisation of technical requirements for registration of pharmaceuticals for human use 1994. Available from https://www.ich.org/ fileadmin/Public_Web_Site/ICH_Products / Guidelines / Efficacy / E1 /Step4 / E1 Guideline.pdf.

80. Lyketsos, C.G., et al., Neuropsychiatric symptoms in Alzheimer's disease. Alzheimer's \& Dementia : the journal of the Alzheimer's Association, 2011. 7(5): p. 532-539.

81. Knuff, A., et al., Use of Actigraphy to Measure Symptoms of Agitation in Dementia. The American Journal of Geriatric Psychiatry, 2019.

82. Rosenberg, P.B., S.K. Wanigatunga, and A.P. Spira, The Potential of Actigraphy to Assess Agitation in Dementia. Am J Geriatr Psychiatry, 2019.

83. European Medicines Agency, ICH E9 (R1) addendum on estimands and sensitivity analysis in clinical trials to the guideline on statistical principles for clinical trials. 2017. 